\title{
Monolayer studies of GHK hydrophobic derivatives
}

\author{
F. Reig ${ }^{1}$, P. Sospedra ${ }^{2}$, N. Almiñana ${ }^{1}$ and A. Ortiz ${ }^{2}$ \\ ${ }^{1}$ Department of Peptide and Protein Chemistry, Institute for Chemical and \\ Environmental Research, CSIC, Jordi Girona 18, 08034 Barcelona, Spain \\ ${ }^{2}$ Department of Physicochemistry, Faculty of Pharmacy, University of Barcelona, \\ Pza. Pius XII, s/n, 08028 Barcelona, Spain
}

\begin{abstract}
A peptide sequence involved in collagenase activity has been synthesized as well as its hydrophobic derivatives. The physicochemical properties of the three corresponding peptide analogues are described. Results indicate that decanoyl derivative is the most active as surface activity and monomolecular layer formation are concerned.
\end{abstract}

\section{INTRODUCTION}

It is a common practice with active peptides to derivatize them hydrophobically in order to improve either their activity or at least their affinity for biomembranes [1-2]. GHK sequence has been described as collagenase inhibitor and could potentially be useful in the cosmetic field. Nevertheless, its small size and high hydrophilicity possess a lot of practical problems concerning its biological activity. In order to improve its physicochemical properties, three hydrophobic derivatives have been synthesized, and their interactions with a mixture of stratum corneum (SC) lipids studied.

\section{EXPERIMENTAL SECTION}

\subsection{Synthesis}

The synthesis of parent peptide and its hydrophobic derivatives was carried out on solid phase using a CLEAR type resin. Couplings were performed with diisopropylcarbodiimide (DIPCDI) and hydroxybenzotriazol (HOBT). Cleavage was performed with TFA/water treatment (9:1) for 3 hours. Peptides were characterized by HPLC and mass spectrometry.

\subsection{Monomolecular layer studies}

\subsubsection{Surface activity}

Surface activity was measured on a NIMA technology Langmuir film balance equipped with a Wilhemy plate, a pression sensor PS4, a ST 9000 tensiometer and a software trough. These experiments were performed in a circular Teflon cuvette $40 \mathrm{ml}$ capacity. Peptides were dissolved in DMSO and injected into the PBS subphase $(\mathrm{pH}=7.4)$, through a small hole drilled at an angle in the wall. The surface pressure increases were recorded as a function of time. The subphase was continuously stirred and the temperature of the system was $21+-1^{\circ} \mathrm{C}$.

\subsubsection{Compression isotherms}

Surface pressure-area isotherms were measured with the same control device but using a rectangular Teflon cuvette of surface area $525 \mathrm{~cm}^{2}$ and volume $240 \mathrm{~cm}^{3}$. The barrier compressed the monolayer at a speed of $50 \mathrm{~cm}^{2} / \mathrm{min}$ (symetrical compression). Peptide solutions were spread from DMSO solutions and 
10 min were left before starting the compression process. The films were compressed up to their collapse pressure. All runs were carried out in triplicate.

The interaction of these peptides with stratum corneum lipids was determined through the compression isotherms of their binary mixtures [3].

\section{RESULTS AND DISCUSSION}

\subsection{Synthesis}

Peptide sequences synthesized were: Hexanoyl-GHK (H-GHK), Decanoyl-GHK (D-GHK) and MyristoylGHK (M-GHK). Yields were higher than $90 \%$.

Mass-spectra agreed with the chemical formula and HPLC profiles indicated that the purity based on peak area was higher than $95 \%$. Before use, peptides were washed extensively with hexane in order to remove traces of hydrophobic impurities. Table 1 shows molecular mass and HPLC data.

Table 1: Analytical data of Synthetic Peptides.

\begin{tabular}{|l|l|l|}
\hline Peptide & Retention Time* & ESI-MS[ $\left.{ }^{+}\right]$ \\
\hline H-GHK & 12.90 & 438.6 \\
\hline D-GHK & 12.88 & 495.2 \\
\hline M-GHK & 12.90 & 550.78 \\
\hline
\end{tabular}

*Elution conditions: Gradient 20 to $95 \%$ of $\mathrm{ACN}+0.05 \% \mathrm{TFA}$. C-18 column.

\subsection{Surface Activity}

The surface activity was determined by injecting different volumes of concentrated DMSO solutions of the peptides into the PBS subphase and recording the pressure increases. The lack of activity of DMSO was determined previously.

The incorporation of the peptides to the air/water interface was similar for all of them. After $30 \mathrm{~min}$ surface pressure was almost constant. Nevertheless all the experiments were carried out for one hour. Figure 1 shows the surface behavior of these peptides in the concentration range under study.

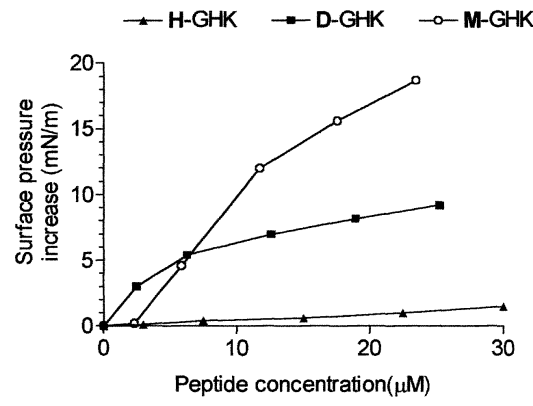

Figure 1: Surface activity of the three peptides derivatives.

Myristoyl derivative was the most active in agreement with the largest length of the acyl chain. Pressure increases were important, for peptides of this size, indicating a clear tendency of M and D-GHK molecules to locate at the air/water interface. 


\subsection{Compression Isotherms}

Compression isotherms of these peptides are given in Fig. 2. H-GHK and M-GHK form monolayers with low collapse pressures and area/molecule values. By contrast, D-GHK monolayer appears to be stable on compression, being its collapse pressure $(16 \mathrm{mN} / \mathrm{m})$, unusually high for molecules of this size. Extrapolated area/molecule values $\left(0.68 \mathrm{~nm}^{2} /\right.$ molec. $)$, correspond to a $\alpha$-sheet.

Mixtures of these peptides with SC lipids (30\% molar), form stable monolayers with collapse pressures equal to that of pure SC monolayers.(Fig 2). Comparing the three mixed isotherms with those of pure components one can appreciate that mixed monolayers have intermediate values.

This indicates that although these peptides are not very active by themselves, once mixed with other lipid components are able to remain associated to them in the monolayer all over the compression process. D-GHK is the one giving more expanded monolayers, suggesting a better association with CS lipids. Besides, the phase change detected for pure D-GHK is retained, although appear at higher surface pressure.

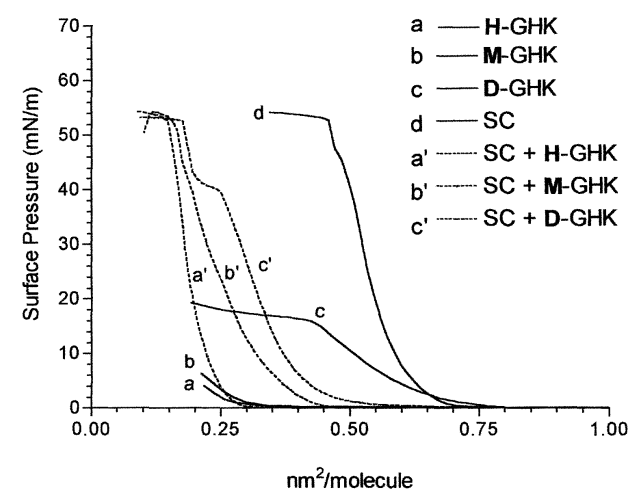

Figure 2: Compression isotherms:a, b, c, d, pure components; a', b',c' mixed monolayers.

\section{Conclusions}

As a summary, Decanoyl-GHK peptide is the molecule that better interacts with lipid monolayers. Its insertion in SC ordered arrays has a soft stabilizing effect. For these reasons it seems to be the most suitable derivative for a further association of this peptide to liposomes.

\section{References}

1.- T. Tachi, R. F. Epand, R. M. Epand, K. Matsuzaki, Biochemistry 41, 10723 (2002).

2.- R. Renthal and D. Velasquez, J. Prot. Chem. 21(4), 255 (2002).

3.- N. Almiñana, M.A. Alsina, M. Espina, F. Reig, J. Coll. Interf. Sci. (in press) (2003).

\section{Acknowledgments}

The technical assistance of Anna Bonet and Maria Osuna is highly acknowledged. N. Almiñana was recipient of a grant from the Ministerio Ciencia y Tecnología. This work was financed with a grant SAF-97-0174. 